\title{
Hysteresis loops of Cosmic Ray intensity decreases versus solar and interplanetary parameters
}

\author{
R. P. Kane \\ Instituto Nacional de Pesquisas Espaciais - INPE, Caixa Postal 515, 12245-970, São José dos Campos, SP, Brazil
}

Received: 29 March 2007 - Revised: 11 September 2007 - Accepted: 14 September 2007 - Published: 2 October 2007

\begin{abstract}
The purpose of this study was to examine the correlation between CR (Cosmic Ray) intensity and solar, interplanetary and terrestrial parameters. The hysteresis loops of (CR) versus those of several solar parameters showed narrow loops in even cycles 20, 22 and broad loops in odd cycles 19,21 , as also in the recent odd cycle 23. Hysteresis plots for $\mathrm{CR}$ versus interplanetary number density $\mathrm{N}$ and speed $\mathrm{V}$ were erratic and uncertain (broad and narrow, all mixed up). Plots of CR versus Interplanetary magnetic field (IMF) $B$ seemed to be narrow for even as well as odd cycles. Hysteresis loops between $\mathrm{CR}$ and other interplanetary parameters were not clear-cut. The same was true for terrestrial parameters. During sunspot maximum years 2000-2003 of cycle 23, there is a double peak structure in all parameters. Whereas CR have a peak spacing of $\sim 30$ months, sunspots and Tilt angle have a spacing of only $\sim 20$ months. The solar open magnetic flux and the Voyager 1 magnetic field have a spacing of $\sim 25$ months. The solar polar magnetic field reverses later than the first peak of all parameters and hence, could not be a direct cause (as if effect started before the cause and lasted for several months more after the cause disappeared). It seems that CR modulation is mainly guided by magnetic configurations deep in the heliosphere, which may not have a simple relationship with parameters near Earth or near Sun.
\end{abstract}

Keywords. Interplanetary physics (Cosmic rays; Heliopause and solar wind termination; Interplanetary magnetic fields)

\section{Introduction}

Cosmic ray (CR) intensity shows variations on several time scales. Forbush decreases occur in a matter of days. Then, there are 27-day variations. On a longer time scale, there

Correspondence to: R. P. Kane

(kane@dge.inpe.br) is a year-to-year variation almost anti-parallel to the 11-year sunspot cycle, but with differences in the even and odd cycles (22-year modulation). The CR modulation starts with a delay with respect to sunspots and the delay is larger in odd cycles $(19,21)$ as compared to the delay in even cycles $(20,22)$. The mechanism for CR modulation consists of time-dependent heliospheric drifts and outward propagating diffusive barriers, which are formed by merging of coronal mass ejections (CMEs), shocks and high-speed flows at 10-15 AU from the Sun (Merged Interaction Regions, MIRs; Burlaga et al., 1985). Since only some MIRs are effective in modulating CRs throughout the heliosphere (Burlaga et al., 1993), Global MIR (GMIR) were conceived which are regions extending $360^{\circ}$ around the Sun mostly in the ecliptic plane and responsible for the step-like changes in CR counting rates. The convection-diffusion mechanism is independent of the sign of the solar magnetic field and operates similarly in every 11-year sunspot cycle (Dorman, 1959; Parker, 1963, and others). On the other hand, the drift mechanism gives opposite effects with the changing sign of the solar magnetic field in alternate cycles (Jokipii and Davila, 1981; Jokipii and Thomas, 1981; Lee and Fisk, 1981; Potgieter and Moraal, 1985, and many other further papers). At the sunspot maximum of odd cycles, the solar north-polar magnetic field reverses, from outward directed $(A>0)$ to inward directed $(A<0)$ during an interval of a few months. A few months later, the solar south-polar magnetic field also reverses, from inward directed $(A<0)$ to outward directed $(A>0)$ during an interval of a few months. In even cycles, the opposite occurs. In $A>0$ epochs, the inflows of CR into the inner heliosphere are faster from over the poles than from along the heliospheric current. When $A<0$, the opposite occurs (Wibberenz et al., 2002). Cane et al. (1999) have reported a very good anti-correlation between CR changes and interplanetary total magnetic field $\boldsymbol{B}$ for two consecutive cycles 21 and 22, and a very close relationship between CR and Tilt angles (latitudinal extent of the heliospheric current sheet HCS, Hoeksema,

Published by Copernicus Publications on behalf of the European Geosciences Union. 


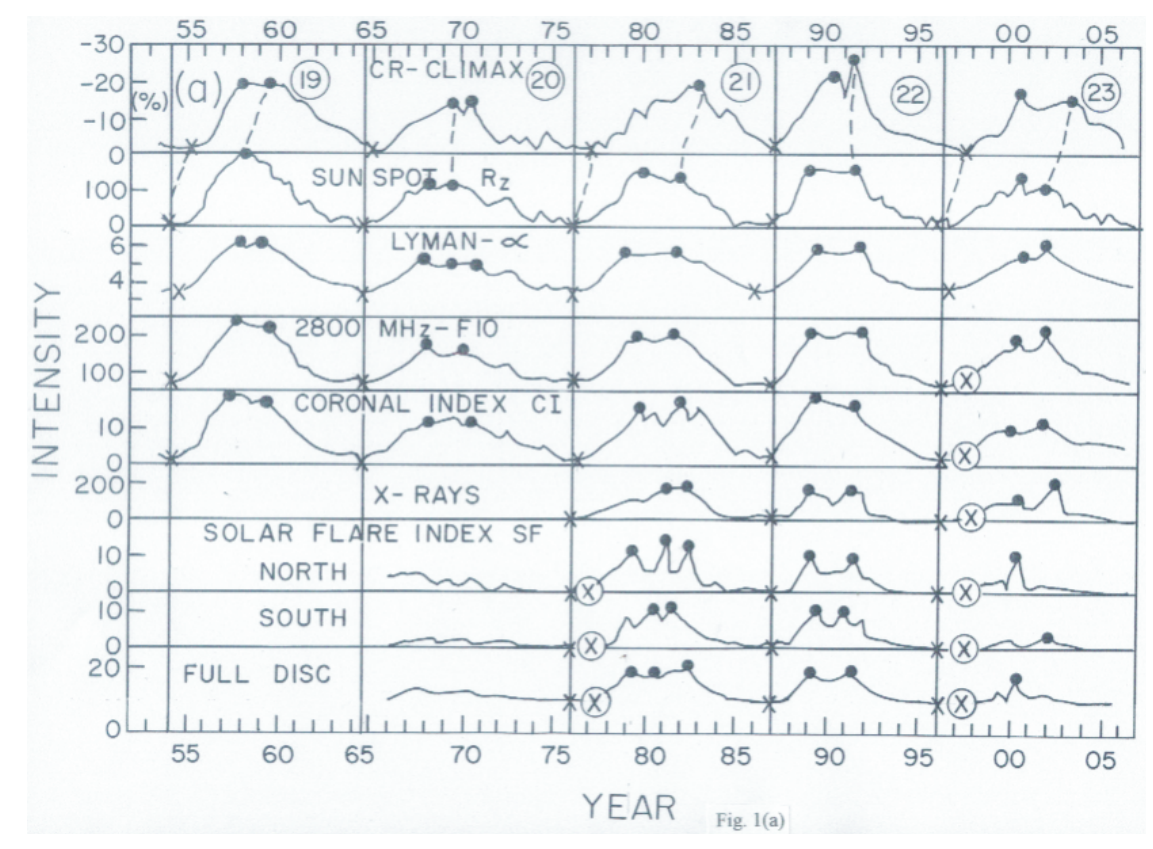

Fig. 1a. Plots of 3-monthly values of solar parameters.for cycles 19-23 (1954-2006). Ordinate (intensity) scale is arbitrary, adjusted to economize the available space. Top plot: CR (Clímax neutron monitor) counting rates as percentage deviations from a $100 \%$ level, plotted upside down. Prominent maxima are marked with big dots and minima with crosses. Further plots are for several solar parameters, namely, sunspot number $R z$ (vertical lines mark sunspot mínima of the various cycles), Lyman- $\alpha, 2800 \mathrm{MHz}(10.7 \mathrm{~cm})$ solar radio emission F10, coronal green line emission index (CI), X-ray background intensity, Solar Flare Index SF for the Northern (N) and Southern (S) Hemispheres of the Sun and for their sum $\mathrm{N}+\mathrm{S}$.

1995; http://wso.stanford.edu/Tilts.html) of the wavy current sheet in the heliosphere, which has been claimed to be very successful as a proxy for solar activity in cosmic ray modulation models with particle drifts included. (Ferreira and Potgieter, 2002). It may be noted that the HCS tilt angle is considered to be a good proxy of solar activity in even cycles during low to moderate modulation conditions (tilt angles below $35-40^{\circ}$ ), while for higher solar activity and for odd cycles, the tilt angle dependence is not so clear. Also, there are two methods to define the HCS tilt angle, the radial model and the average $L_{a v}$. Here we have used $L_{a v}$.

The delays (different in odd and even cycles) are displayed in hysteresis plots of $\mathrm{CR}$ versus sunspots, where the hysteresis loops are broad in odd cycles and narrow in even cycles (Dorman, 2001; Dorman et al., 2001a, b, and references therein). Presently, cycle 23 is coming to its end, and it would be of interest to check whether the hysteresis loop was broad in this odd cycle 23 .

In the present communication, the hysteresis plots are shown for CR versus sunspots as also versus some other solar parameters, and further for CR versus interplanetary and terrestrial parameters.

\section{Plots}

Solar data have been obtained from the NOAA website http: //www.ngdc.noaa.gov/stp/SOLAR/solintro.html and other data from the website http://cdaw.gsfc.nasa.gov/geomag cdaw/Data.html. Figures 1a, b, c show the plots of 3-monthly values of several parameters for cycles 19-23 (1953-2006). (The ordinate intensity units are arbitrary, set to use the space economically). In Fig. 1a, the top plot is for CR (Clímax neutron monitor) counting rates as percentage deviations from a $100 \%$ level, plotted upside down so that CR maximum depressions match with sunspot maxima. Prominent maxima are marked with big dots and minima with crosses. If there is a prominent maximum and another maximum of almost the same magnitude, both (or even three) are shown with big dots (this is, of course, highly subjective, and opinions may differ). The next plot is for sunspot number (vertical lines mark sunspot mínima of the various cycles). As can be seen, in odd cycles $19,21,23$, the CR maxima and minima occur with a substantial delay with respect to the sunspot maxima and minima (shown by the inclined dashed lines joining the CR plot to the $R z$ plot). Further plots are for solar Lyman- $\alpha(121.6 \mathrm{~nm}$, a chromospheric emission, Woods et al., 2000, updated), $2800 \mathrm{MHz}(10.7 \mathrm{~cm})$ solar radio emission F10 (origin mostly in lower corona), the coronal green line $(530.3 \mathrm{~nm})$ emission index CI (Rybansky et al., 2005) and X-ray background intensity (mostly in the corona). In 


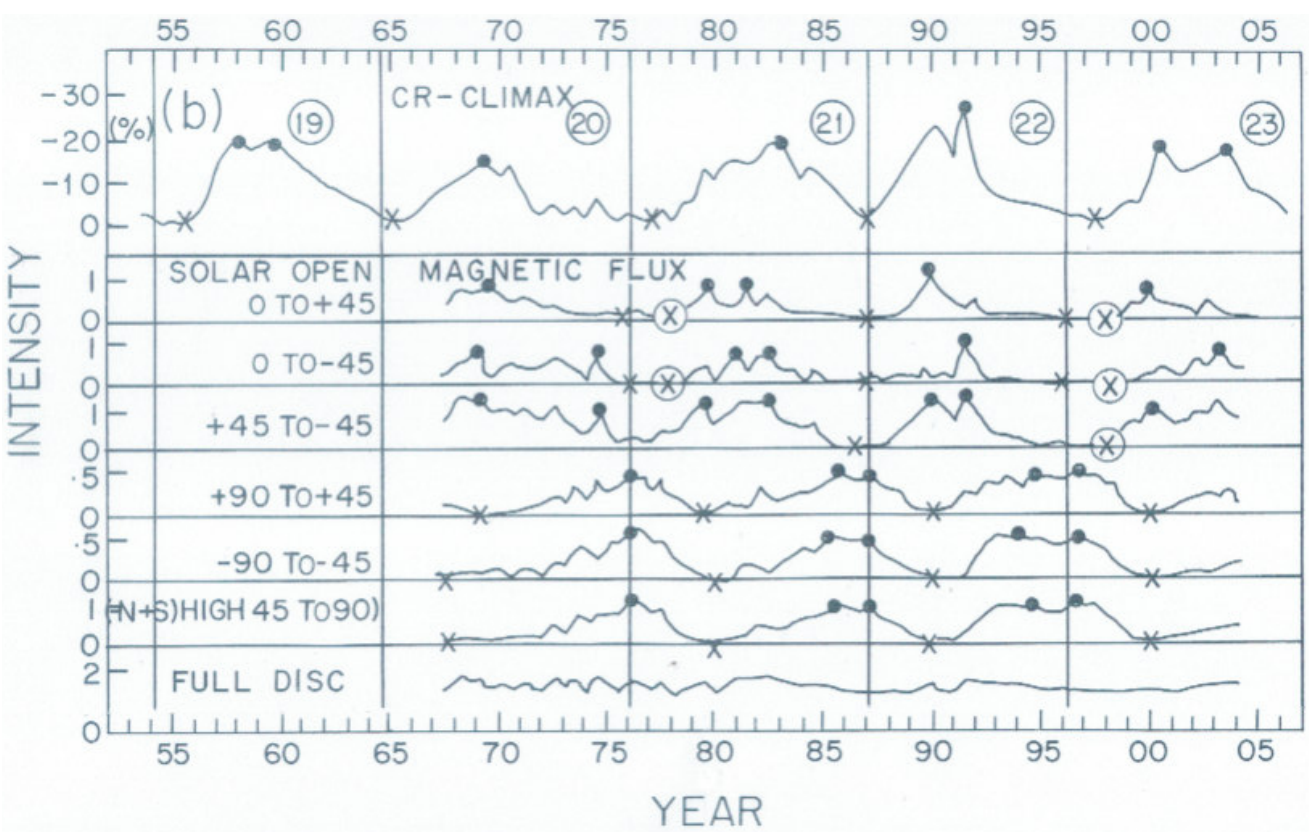

Fig. 1b. Top plot: CR (Clímax neutron monitor, repeated). Further plots are for open magnetic field fluxes, for low and high solar latitudes.

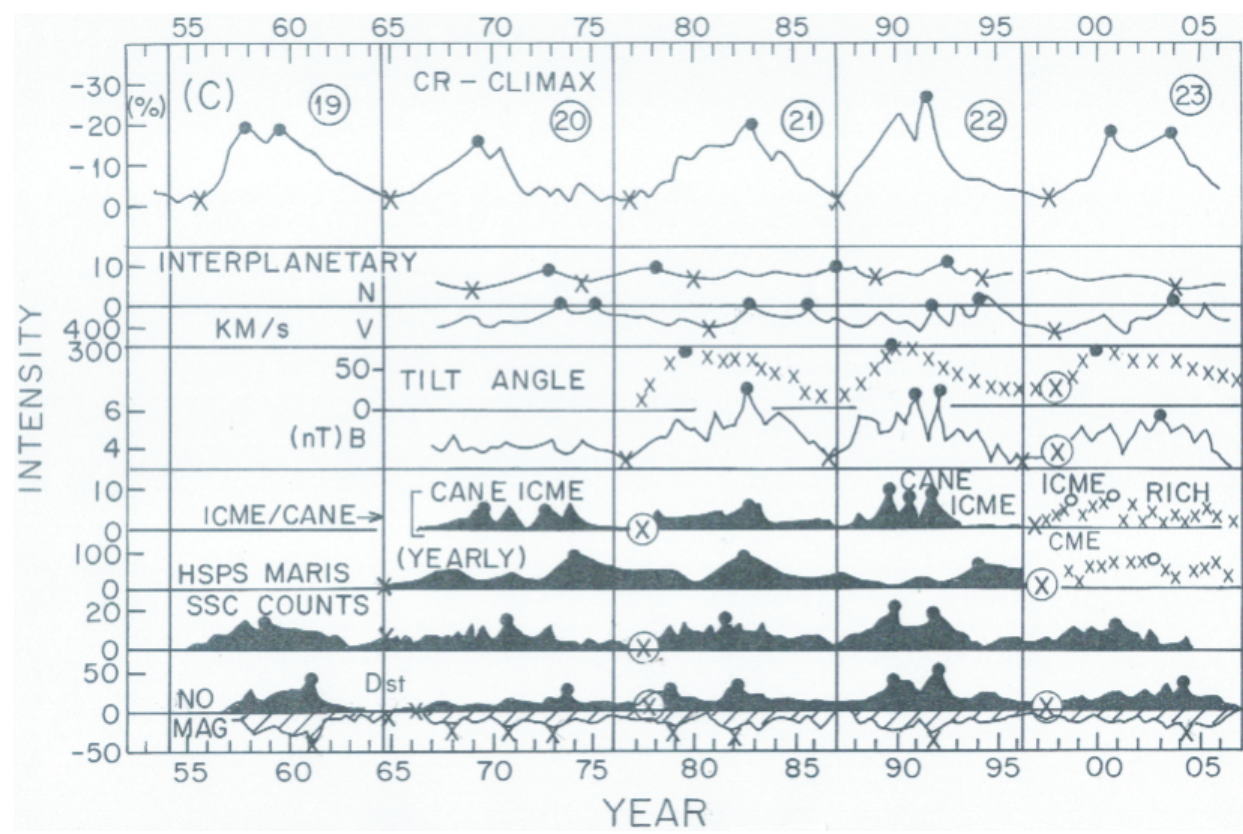

Fig. 1c. Top plot: CR (Clímax neutron monitor, repeated). Further plots are for interplanetary parameters, namely, number density $N$, solar wind speed $V$ and total magnetic field $\boldsymbol{B}$, Tilt angles (crosses), CMEs and ICMEs, HSPS (high-speed plasma streams), and terrestrial parameters, namely, occurrence frequency of Storm Sudden Commencement events, occurrence frequency of the geomagnetic disturbance index $D_{s t}$ events of negative magnitudes exceeding $50 \mathrm{nT}$, and the average 3-monthly magnitudes of $D_{s t}$.

cycle 19 , one can see two CR peaks, only one sunspot peak, two Lyman- $\alpha$ peaks, two $2800 \mathrm{MHz}$ radio emission peaks and two peaks in the coronal index. In cycle 20 , one can see two peaks in CR and sunspots, possibly three peaks in Lyman- $\alpha$, and two peaks each in $2800 \mathrm{MHz}$ and coronal index. In cycle 21 , there is only one strong peak in CR, but two peaks in the solar radiations. In cycle 22 and 23, there are two maxima in all these parameters. In general, solar parameters show variations almost similar to those of sunspots (matching within a few months), including the two 
prominent maxima near sunspot maximum. Further plots are for the Solar Flare Index SF (Atac and Ozguc, 1998, updated) for the Northern (N) and Southern (S) Hemispheres of the Sun and for their sum N+S. These plots are somewhat different from the plots of sunspots, notably the structure at sunspot maximum.

The solar photosphere has substantial magnetic field structures. These are mostly closed, but sometimes, there are open magnetic regions (coronal holes) on the Sun, which transmit magnetic fields to the interplanetary space (Wang et al., 2000; Wang and Sheeley, 2002). Figure $1 \mathrm{~b}$ shows the plots for CR intensity (repeated) and for the open magnetic fields. The top plot for CR (Clímax neutron monitor) counting rates is the same as in Fig. 1a. Further plots are for the open magnetic fields. Three plots are shown, for low solar latitudes North $\mathrm{N}(+)$ and South $\mathrm{S}(-)\left(0\right.$ to $+45^{\circ}, 0$ to $-45^{\circ}$ and their sum $+45^{\circ}$ to $\left.-45^{\circ}\right)$. These have variations almost similar to sunspot activity (discussed in detail in Kane, 2006a), with slight differences for the maxima. Next three plots are for high solar latitudes North $\mathrm{N}(+)$ and South $\mathrm{S}(-)\left(+90^{\circ}\right.$ to $+45^{\circ},-90^{\circ}$ to $-45^{\circ}$ and their sum $\left(\mathrm{N}+\mathrm{S}\right.$ high latitude $45^{\circ}$ to $90^{\circ}$ ). Here, the variations are quite different, almost antiparallel to sunspot activity; the maxima are during sunspot minimum (vertical lines). Thus, the solar open magnetic flux leaving the solar atmosphere has very different characteristics at low and high solar latitudes.

Figure 1c shows plots for CR intensity (repeated) and interplanetary parameters, namely, number density $\mathrm{N}$ (mostly protons), solar wind speed $\mathrm{V}$ and total magnetic field $\boldsymbol{B}$ (daily values of $B_{x}, B_{y}, B_{z}$ used for calculating daily $\boldsymbol{B}$, used further for obtaining average 3-monthly $\boldsymbol{B}$ ), for cycles 20 23 (no data for cycle 19). Here, the number density $N$ shows variations unrelated to sunspot activity; maxima appear irregularly, at different phases of sunspot activity. The solar wind speed $V$ shows maxima mostly during the declining phases of sunspot activity. The total magnetic field $\boldsymbol{B}$ is very low throughout cycle 20 , but for cycles $21-23, B$ shows maxima similar to the sunspot numbers. The crosses show a plot of the Tilt angles for cycles 21, 22, 23 and the plot is very similar to the plots of sunspots.

Some data about CME, obtained from various sources, are also plotted. Cane et al. (1996) compiled a list of ICMEs (Interplanetary CMEs near the Earth) which caused CR decreases exceeding 4\%, during 1966-1996 (cycles 20-22). Thus, the plot (CANE ICME) is only for a part of the total ICMEs near Earth. The variation is roughly parallel to sunspots, but statistics seems poor. For cycle 23 (1996 onwards), I. G. Richardson (private communication, 2006) provided us a total ICME list (from satellite data). The variation is roughly parallel to sunspots. Maris and Maris (2003) provided a list of HSPS (high-speed plasma streams) for 19661996 (cycles 20-22). The maxima seem to occur in the declining phase of sunspot activity, probably because highspeed corotating streams occur more during the declining phases. For cycle 23, CME frequency (crosses in the third plot from bottom in the last column) was available from the SOHO LASCO CME catalogue (http://cdaw.gsfc.nasa. gov/CME_list/). These show variations roughly parallel to sunspots.

Some terrestrial parameters can be used as indicators of interplanetary disturbances. Three parameters are plotted in Fig. 1c, bottom part, for cycles 19-23. These are, the Occurrence frequency of Storm Sudden Commencement (SSC) events, the Occurrence frequency of the geomagnetic disturbance index $D_{s t}$ events of negative magnitudes exceeding $50 \mathrm{nT}$, and the average 3-monthly magnitudes of $D_{s t}$. All these show variations roughly parallel to sunspots.

Since in odd cycles, there is considerable delay in the commencement of CR modulation, it would be interesting to see which parameters have minima coinciding with sunspots and which show delays similar to those of CR. For the odd cycle 19 where CR lagged considerably behind sunspots $R z$ (the tilted dashed line joining the two in Fig. 1a), Lyman$\alpha, 2800 \mathrm{MHz}$, coronal index show mínima matching with sunspots. For the even cycle 20, CR lags were almost nil and the parameters Lyman- $\alpha, 2800 \mathrm{MHz}$, coronal index as well as the terrestrial parameters had minima coinciding with sunspots (and with CR also). For the odd cycle 21, CR had a considerable lag with respect to sunspots (the tilted dashed line joining the two), while Lyman- $\alpha, 2800 \mathrm{MHz}$, coronal index, and X-rays had minima coinciding with sunspot minimum (no lags, all at the vertical line). On the other hand, the solar flare index (Fig. 1a) and the solar open magnetic flux at low solar latitude (Fig. 1b) show lags (increases starting further away from the vertical line) so that the minima coincide with CR (marked as encircled crosses). The SSC counts and $D_{s t}$ (Fig. 1c) also show similar lags, but the IMF $B$ does not show the lag. This is surprising as the lags are considered as indicative of effective regions taking time to reach deep into the heliosphere. How then parameters near the Sun (open magnetic flux) or even near the Earth (1 $\mathrm{AU}, D_{s t}$ etc.) show a lag? For the even cycle 22, CR showed no lags. All other parameters also had no lags (minima coincided with sunspot minima). For the odd cycle 23, CR lagged considerably behind sunspots. However, many other parameters also show lags (circled crosses, away from the vertical line of sunspot minimum). Thus, the interpretation of lags as delays due to effective regions taking time to reach deep in the heliosphere needs reconsideration and further scrutiny.

Figure 2 shows the hysteresis plots for CR versus sunspot number $R z$ (at the photosphere) in the first column, for the five successive cycles 19 (1953-1964), 20 (1964-1976), 21 (1976-1987), 22 (1987-1996) and 23 (1996-2006). The full lines are for the rising phase of the solar cycle and the crosses for the declining phase. As can be seen, the hysteresis loops are broad in the odd cycles 19,21 and 23 and narrow in the even cycles 20 and 22. Thus, for sunspots, cycle 23 has conformed to the general pattern of broad odd cycles. The second column is for solar Lyman- $\alpha$. Here, the patterns are similar to those for sunspots, except that for cycle 23 , 


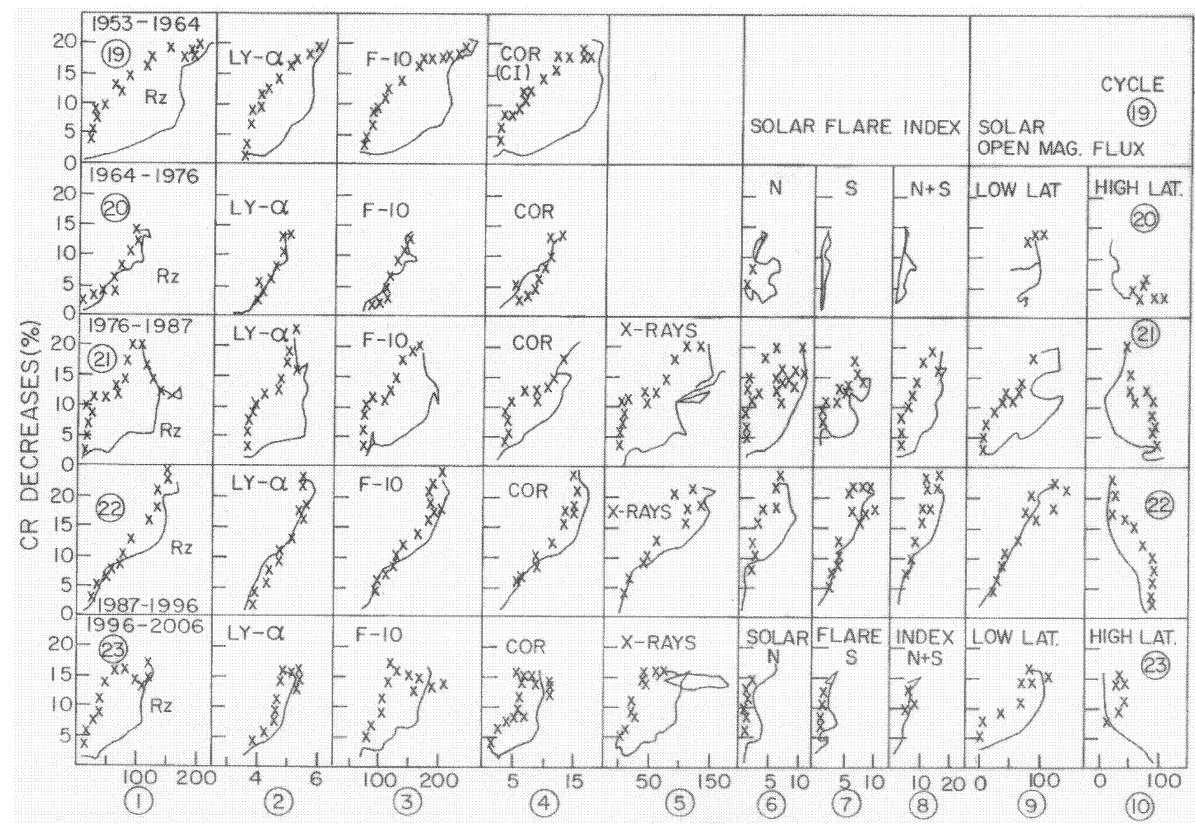

Fig. 2. Hysteresis plots of Cosmic Ray (CR) intensity (ordinate in percent, 3-monthly values) versus several solar parameters (abscissa), columns 1-10. The abscissa scale is arbitrary, adjusted to suite the available space. The full lines are for the rising part of the solar cycle and the crosses for the declining part.

the pattern is not very broad (reason not known). The third column refers to the $2800 \mathrm{MHz}(10.7 \mathrm{~cm})$ solar radio emission F10. The patterns are very similar to those of sunspots. The fourth column refers to the coronal green line emission and shows patterns almost similar to those of sunspots and F10. Thus, for solar parameters originating in a wide range of solar altitudes, the hysteresis patterns are mostly similar, though small differences exist. The fifth column refers to Xray background intensity (for cycles 21,22 and 23 only), and the patterns conform roughly to broad hysteresis loops in the odd cycles 21, 23 and narrow loops in the even cycle 22 . The sixth and seventh columns are for the Solar Flare Index SF for the Northern $(\mathrm{N})$ and Southern (S) Hemispheres of the Sun. For cycle 20, the data for SF (N) show a broad loop and data for (S) show a narrow loop (the sum $\mathrm{N}+\mathrm{S}$ in the eighth column shows a narrow loop), but the data quality is suspect. For cycle 21, the loops are broad as expected for an odd cycle. For cycle 22, the loop for $\mathrm{N}$ is broad (even cycle, not expected); but the loops for $\mathrm{S}$ and $\mathrm{N}+\mathrm{S}$ are narrow (expected). However, for cycle 23 , all $\mathrm{N}, \mathrm{S}$, and $\mathrm{N}+\mathrm{S}$ loops are narrow (odd cycle, not expected). Thus, the hysteresis patterns of the solar flare index (coronal phenomenon) did not always conform to expected patterns, probably because solar flares are transient phenomena with irregular occurrences.

The ninth column shows hysteresis loops for solar open magnetic field flux for low solar latitudes $\left(+45^{\circ}\right.$ to $\left.-45^{\circ}\right)$, which are known to have a 11-year variation parallel to sunspot activity (discussed in Kane, 2006a). Data for cycle 20 are incomplete (data available only for the declining phase), but for cycle 21 and 23, the loops are broad and for cycle 22 , the loop is narrow, as expected. Thus, the low latitude solar magnetic open flux has hysteresis effects similar to those of other solar parameters. The tenth column refers to solar open magnetic flux at high solar latitudes $\left(45^{\circ}\right.$ to $90^{\circ}$, $\mathrm{N}$ and $\mathrm{S}$ ). These fluxes are known to show a 11-year variation anti-parallel to sunspot activity (Kane, 2006a). The loop for cycle 20 is incomplete, that for cycle 21 is broad (expected), that for cycle 22 is not very narrow, and that for cycle 23 is not broad (unexpected). Thus, magnetic flux leaving the solar atmosphere at low latitudes conforms to the general pattern, but the flux leaving the high solar latitudes (including polar regions) may have different characteristics.

The broad hysteresis loops in the odd cycles 19, 21, 23 and narrow loops in cycles 20,22 could be due to fixed delays (different for every cycle) or due to different evolutions of CR and sunspots at every sunspot phase, or both. To evaluate the average delays, a cross-correlation analysis was carried out between CR and sunspots (with delays in units of $3,6, \ldots 30$ months) separately for all cycles 19-23. Figure 3 shows the results. The best correlations are obtained at average delays of 9 months, 3 months, 9 months, 3 months and 15 months for the five cycles 19-23 (since values used are 3-monthly, results are only as multiples of 3). If the CR values are shifted earlier (or sunspot values are shifted later) by these numbers, the hysteresis loops change as shown in Fig. 4 for the odd cycles 19, 21 and 23 only, where delays were large. The full lines are for the rising phase of the solar cycle and the crosses for the declining phase. The left half 


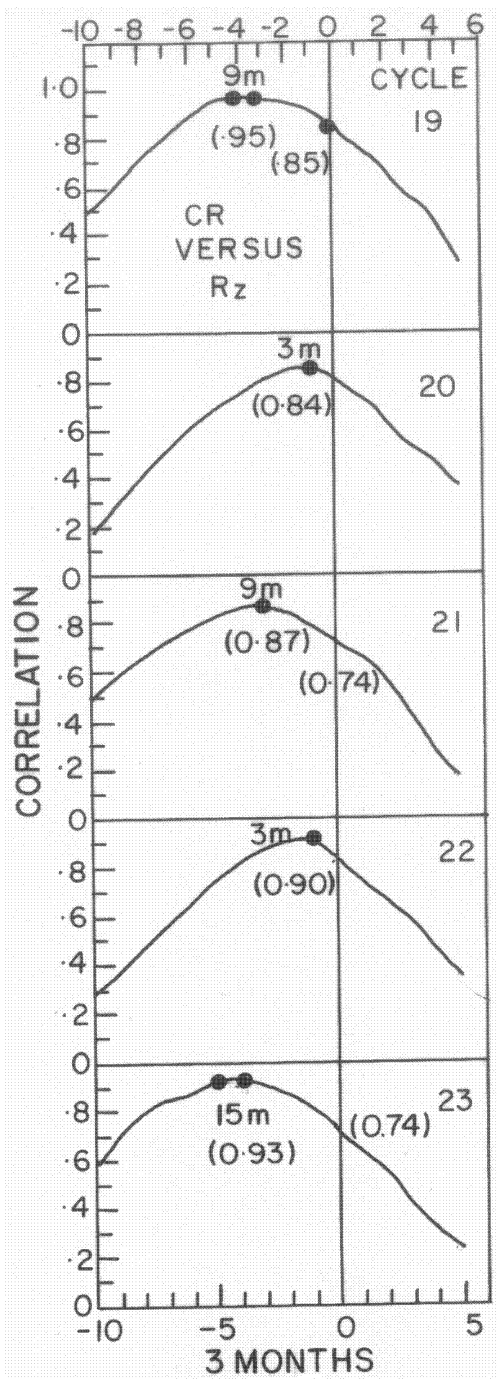

Fig. 3. Cross-correlation between $C R$ and $R z$ for cycles 19-23. Abscissa shows phase difference in 3-month units.

shows the original loops and the right half shows the loops for shifted data. The broad loops become narrow as expected, but some broadening remains. To check whether the solar parameters themselves have a different evolution in odd and even cycles, the long data series of sunspots ( $\sim 250$ years, cycles 1-23) were superposed for about 11 consecutive years, for successive odd $(1,3, \ldots .23)$ and even $(2,4 \ldots .22) \mathrm{cy}-$ cles separately, with the first, starting year being the year of sunspot minimum. The results are shown in Fig. 5. The upper part shows the average sunspot cycle for odd cycles $1,3, \ldots .23$ (full line) and even cycles $2,4, \ldots .22$ ) (crosses) There is slight difference between the two. The lower part shows the deviations from a mean of all cycles (cycles 1-23, mean not shown here) for odd cycles (full line) and even cycles (crosses). It seems that for odd cycles, there is a slight excess of sunspot number in the ascending phase (years 0 7 ), while for even cycles, there is a deficit; but the effect is

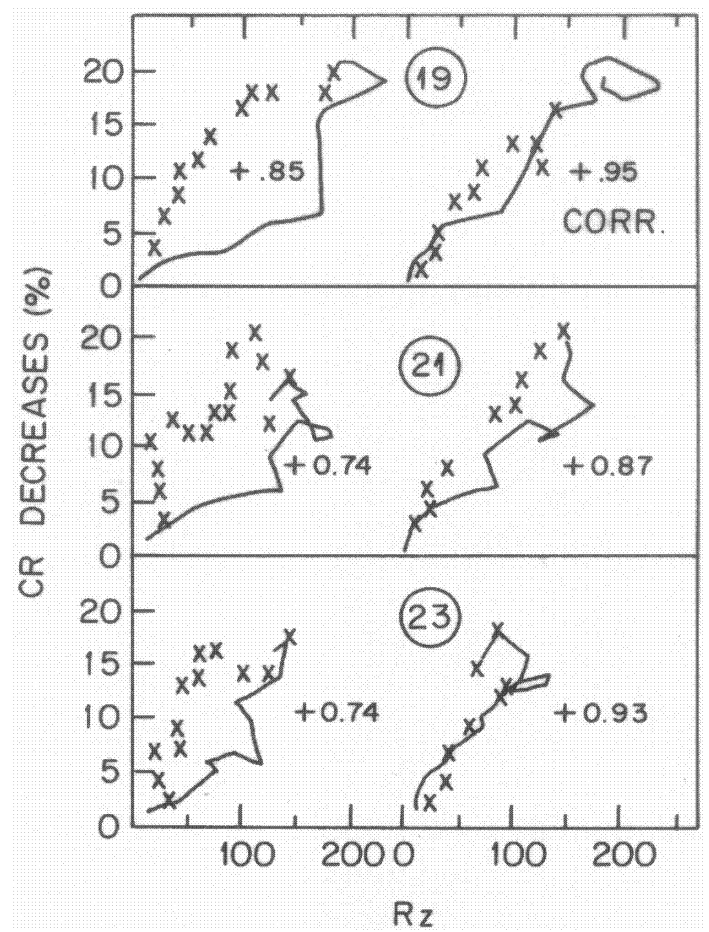

Fig. 4. Hysteresis loops for CR (ordinate in percent) versus $R z$, left half, direct data (original loop), right half $\mathrm{CR}$ values advanced for the estimated delays. The full lines are for the rising part of the solar cycle and the crosses for the declining part.

small, nothing like the behaviour of $\mathrm{CR}$, which is strikingly different in odd and even cycles. In principle, the hysteresis loops of CR versus $R z$ may be affected by two features, firstly, a delay between the starts and secondly, a difference in the evolution of $R z$ in odd and even cycles. The magnitude is $\sim 8$ sunspot number, a difference of $\sim 16$ sunspot numbers $(\sim 16 \%)$ between odd and even cycles. The CR hysteresis of cycle 21 (Fig. 4, left half) shows CR differences of $\sim 10 \%$. Thus, the two may be related partially.

For the interplanetary space, Fig. 6 shows the hysteresis plots of CR versus interplanetary parameters, $N, V, B$. The full lines are for the rising phase of the solar cycle and the crosses for the declining phase. For $N$ in the first column, the loops are erratic, with no clear relationships with odd or even cycles. The same is true for $V$ in the second column. For $B$, Cane et al. (1999) have reported a very good anticorrelation between $\mathrm{CR}$ changes and $B$ for two consecutive cycles 21 and 22, and a very close relationship between CR and Tilt angles of the wavy current sheet in the heliosphere (heliospheric current sheet HCS, data from J. T. Hoeksema, http://wso.stanford.edu/Tilts.html), which has been claimed to be very successful as a proxy for solar activity in cosmic ray modulation models with particle drifts included (Ferreira and Potgieter, 2002). Cane et al. (1999) have shown hysteresis plots of CR versus the Tilt angle. These show broad 


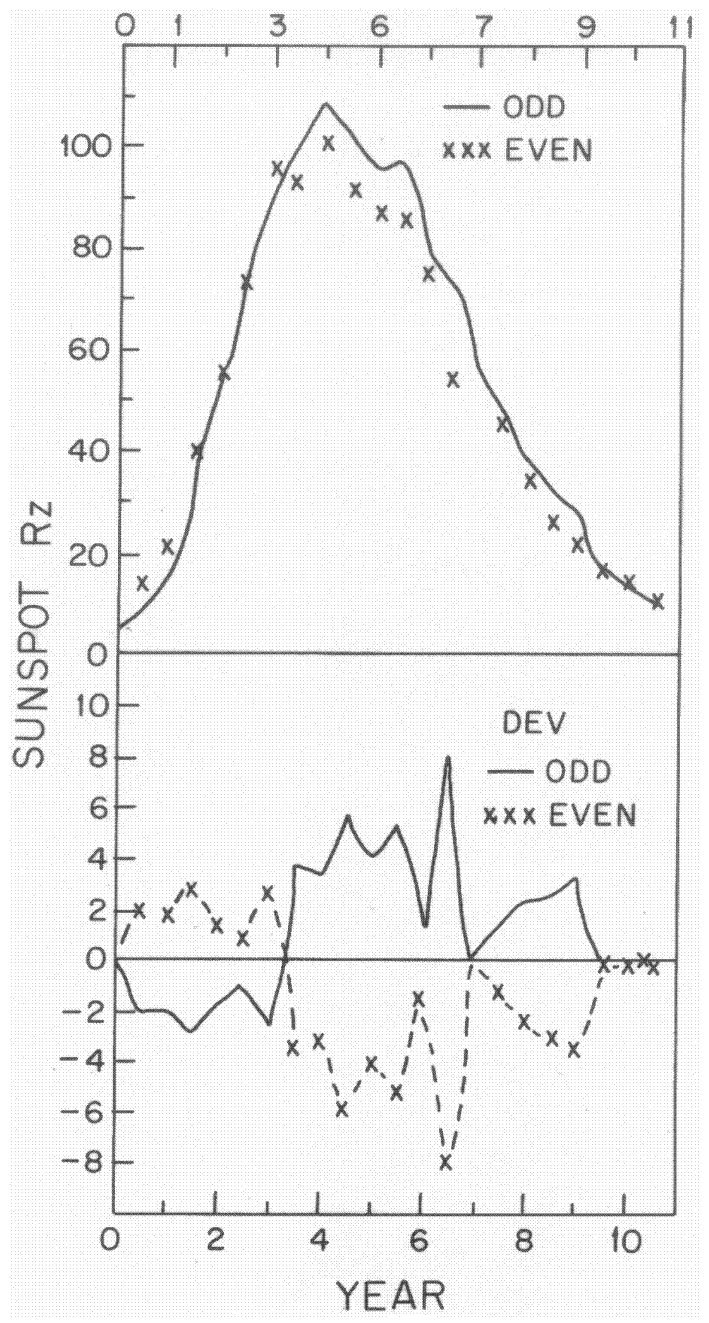

Fig. 5. Upper half, Average sunspot cycles for odd cycles $1,3 \ldots .23$ (full lines) and even cycles 2, 4...22 (crosses). Lower half, deviations of odd and even cycles from the general average of cycles 1 , $2,3 \ldots 23$.

loops in both cycles 21 and 22, but a very good relationship is claimed. In Fig. 6, the third column shows the hysteresis plots for CR versus IMF (interplanetary magnetic field) total component $B$. The loops are partly broad and partly narrow in all the cycles 20-23, indicating a somewhat loose relationship between CR and $B$. The fourth column shows the hysteresis loops for CR versus Tilt angle. The loops are broad in odd cycles 21 and 23 and narrow in the even cycle 22, similar to the loops of CR versus sunspots in Fig. 2, first column. Thus, the Tilt angles have a variation very much parallel to sunspots. The correlations are: $R z$ to Tilt angle $+0.85, R z$ to $\mathrm{CR}+0.79$, CR to Tilt angle +0.70 . The $B$ component has correlations of +0.60 with Tilt angle and $R z$, and +0.70 with CR. Thus, relationship with $B$ is not fully satisfactory.

Figure 7 shows the hysteresis loops for CR versus ICMEs in the first column. The full lines are for the rising phase of

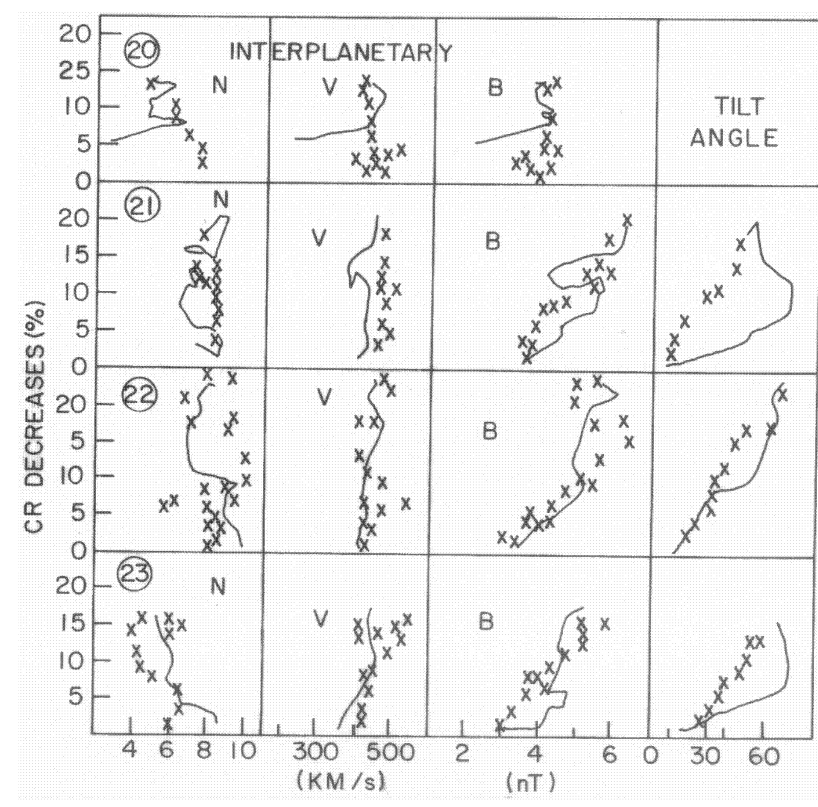

Fig. 6. Hysteresis plots of Cosmic Ray (CR) intensity (ordinate in percent, 3-monthly values) versus interplanetary parameters $N, V$, $B$ and Tilt angle (abscissa, scale arbitrary, adjusted to suite available space).

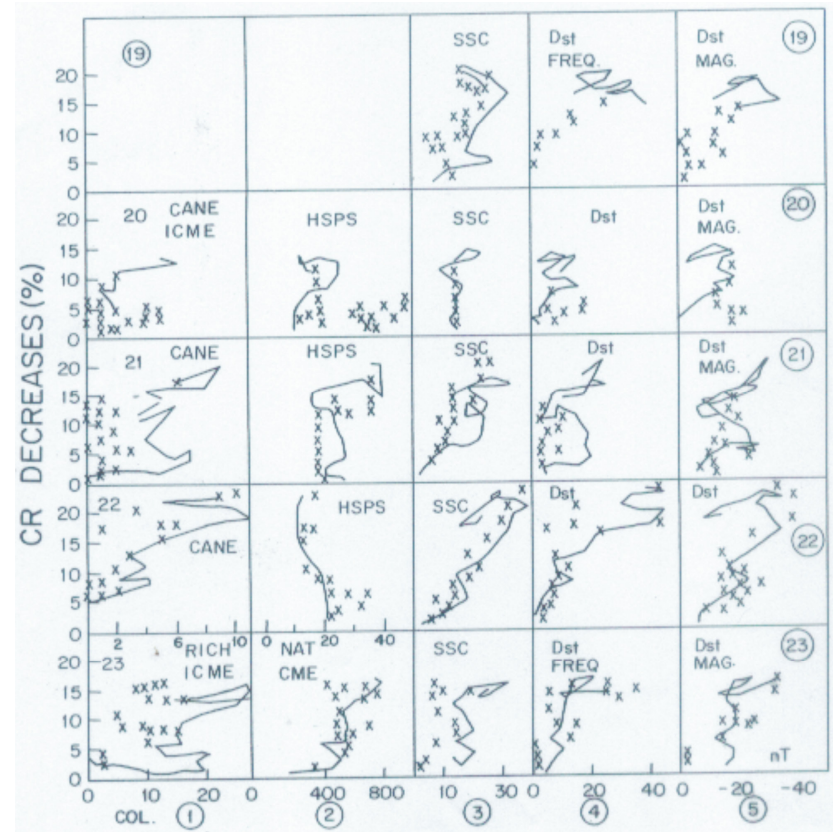

Fig. 7. Hysteresis plots of Cosmic Ray (CR) intensity (ordinate in percent, 3-monthly values) versus CMEs, ICMEs, HSPS, SSC count and $D_{s t}$ occurrence frequencies and magnitudes. The full lines are for the rising part of the solar cycle and the crosses for the declining part.

the solar cycle and the crosses for the declining phase. Loops are broad in odd cycles 21 and 23, but not very narrow in 


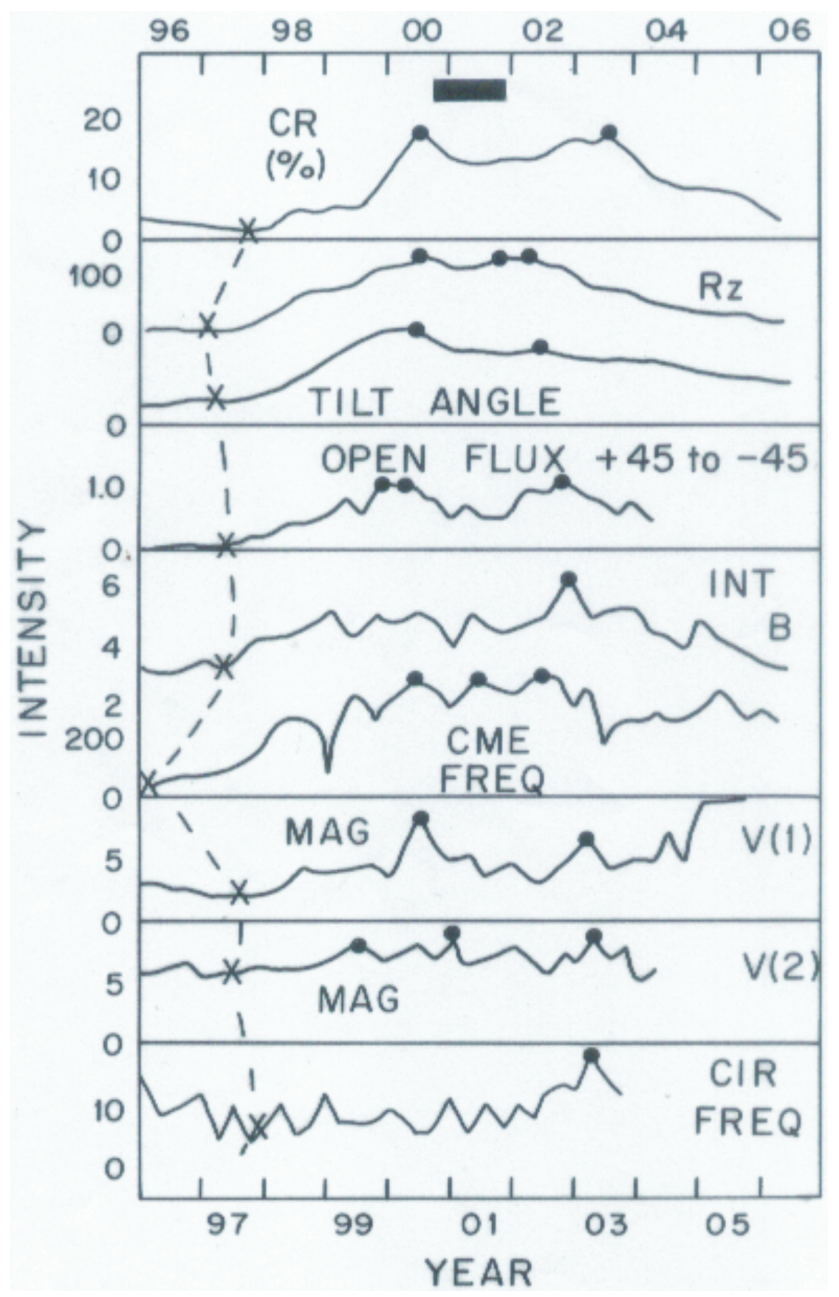

Fig. 8. Plots of 3-monthly values for cycle 23 (1996-2006, ordinate in percent for CR, intensity units arbitrary for others). Maxima are marked with big full dots. Minima during 1996-1997 are marked with crosses. The full rectangle at the top shows the reversal of solar polar magnetic field.

even cycles 20 and 22, partly because the data for ICMEs are scanty (poor statistics). The second column refers to CR versus HSPS (high-speed plasma streams). The plots are erratic with not very broad loops in any cycle. Thus, relationship is obscure. The third column refers to SSC occurrence frequency. Here, there is some indication that loops in even cycles 20, 22 are narrower as compared to loops in the odd cycles 19, 21, 23. Thus, SSC patterns are more like sunspots. The fourth and fifth columns refer to $D_{s t}$ frequencies and magnitudes and do not show any consistent patterns for odd or even cycles. In short, all these terrestrial effects do not have any consistent patterns distinctly different for odd and even cycles.

Figure 8 shows the plots for cycle 23 for some parameters.

1. The top plot is for CR deviations (upside down) and shows two prominent peaks (actually prominent depres- sions, large modulation), one in the middle of 2000 and another in the middle of 2003. The gap between these two peaks is of $\sim 30$ months. The full rectangle at the top shows the reversals of solar polar magnetic fields, in about November 2000 at the north pole and about October 2001 at the south pole (an interval of about one year, Harvey and Resley, 2002). The CR peak occurs earlier than the commencement of magnetic field reversal by several months. So, the reversal could not be a cause of CR changes (effect must follow the cause, not precede it).

2. The next plot for sunspots $R z$ shows one prominent peak in the middle of 2000 (almost matching with CR) and two subsidiary maxima in the end of 2001, almost a year and a half earlier than the second maximum of CR. The $R z$ gap (Gnevyshev gap) is only of $\sim 20$ months, much lesser than the CR gap of $\sim 30$ months. These discrepancies are discussed in detail in Kane (2006b), and some striking features of cycle 23 are discussed in Kane (2006c). The CR modulation started a few (15) months later than solar minimum (see the dashed inclined line joining $\mathrm{CR}$ and $R z$ in Fig. 1a), indicating a delay of solar effects to reach the mid-heliosphere, though sunspots themselves (and other indices like radio noise, Lyman $\alpha$ etc.) do not transmit anything to interplanetary space and hence are, in principle, irrelevant for CR modulation, which occurs in deep heliosphere.

3. The next plot is for Tilt angles and is almost similar to $R z$, except that the second maximum is not very prominent. Physically, tilt angle is in the interplanetary space and hence, certainly relevant for CR modulation, but the spacing here is only $\sim 20$ months (just like that for $R z$ ), and much smaller than the CR spacing (30 months). Incidentally, the first peak of the Tilt angle is almost coincident with (only slightly earlier than) the first peak of CR. So, the lag of several months seen at the starting of CR modulation vis-a-vis sunspot minimum (start of cycle 23) is not seen so much for the first CR peak and Tilt angle peak. So to say, the lag is lost from sunspot minimum to sunspot maximum.

4. The next plot for magnetic open flux (low solar latitudes) has three peaks, two in the beginning of 2000, a few months earlier than the peaks in $R z$ and $C R$ and a third one in 2002 end, later than the second $R z$ maximum but earlier than the CR second maximum by almost one year. The spacing for the open flux is $\sim 25$ months, smaller than the 30 months for $\mathrm{CR}$, but stretching the imagination a bit, one could claim that the open flux could be considered as responsible for a delayed effect for CR modulation.

5. The next plot for IMF $B$ shows only one prominent peak at the end of 2002, a few months earlier than the second 
CR peak, and there is no similarity between variations of $B$ and $\mathrm{CR}$. The next plot of CME frequency shows three peaks, one in the middle of 2000 (same as CR, $R z$ and open flux), a second in the middle of 2001, and a third in the middle of 2002, roughly similar to $R z$ and Tilt angle in spacing etc. The CMEs are an important part of interplanetary space and would affect CR modulation, but the spacing of only $15-20$ months is smaller than the CR spacing of 30 months.

6 . The next plots are for the magnetic fields at Voyager 1 (67-82 AU) and Voyager 2 (52-61 AU) (Burlaga et al., 2001). Voyager 1 had a first peak near the middle of 2000 , about the same as for CR, and a second peak in early to middle of 2003, few months earlier than the CR second maximum. Thus, the magnetic field at Voyager 1 (almost similar to solar open magnetic flux) could be a candidate for CR modulation in a rough way. The Voyager 2 plot has three ill-defined peaks, only the third one coinciding with the second peak of Voyager 1. It may be noted that Voyager 1 was above the ecliptic plane while Voyager 2 was below.

7. The last plot for CIRs (corotating interaction regions, Alves et al., 2006) shows one prominent peak in the middle of 2003, almost matching with the CR second peak. Thus, it seems that magnetic fields deep in the heliosphere, responsible for CR long-term modulation, may have contributions from dissipated CMEs as well as from corotating regions.

\section{Conclusions}

Using data for CR (Cosmic rays) as recorded by the Climax neutron monitor since 1953, the 3-monthly means were compared with those of several solar parameters, namely, sunspots, Lyman- $\alpha, 2800 \mathrm{MHz}$ solar radio emission, coronal green line index, X-rays, Solar flare index, solar open magnetic flux (low solar latitudes), interplanetary parameters, namely, number density $\mathrm{N}$, solar wind speed $\mathrm{V}$ and total magnetic field $\boldsymbol{B}$ and the Tilt angle, CME and ICME counts, and some terrestrial parameters. The following was noted:

1. The hysteresis plots of $\mathrm{CR}$ versus solar parameters showed narrow loops in even cycles 20, 22 and broad loops in odd cycles 19, 21, 23. There was a lag between commencement of CR modulation and sunspot minimum, of about 3 months in even cycles and 9-15 months in the odd cycles. If CR data are adjusted for these lags, the hysteresis loops become narrower but not completely. A comparison of the average 11-year sunspot variations separately for odd and even cycles showed that in odd cycles, $R z$ evolved more rapidly as compared to even cycles. This might have contributed partially to the odd-even difference of CR hysteresis plots.
2. Hysteresis plots for $\mathrm{CR}$ versus interplanetary $\mathrm{N}$ and $\mathrm{V}$ were erratic and uncertain (no clear distinction between patterns for odd or even cycles). Plots of CR versus IMF $B$ were narrow for even as well as odd cycles, indicating a good relationship between CR and $B$, but some discrepancies remained, indicating that this $B$ near Earth may not be fully representative of conditions deep in the heliosphere where CR modulation mainly occurs.

3. The hysteresis loops of CR versus Tilt angles were similar to those of CR versus $R z$, narrow in even cycles and broad in odd cycles. $R z$ has no relevance for interplanetary space and hence for CR modulation. But the Tilt angle is very much relevant.

4. Hysteresis loops between CR and ICMEs or HSPS (high-speed plasma streams) were not clear-cut and no clear distinction was seen between the loops for odd and even cycles. The same was true for terrestrial parameters.

5. A detailed examination for cycle 23 showed the following:

- CR modulation started with a delay of a few months with respect to sunspot minima. This was expected, as cycle 23 is an odd cycle.

- CR had maximum depression (a peak, if vertical scale is upside down) during the high sunspot years 2000-2003. This general anti-correlation between $\mathrm{CR}$ and sunspot number is also expected.

- During the years of sunspot maximum (20002003), $R z$ was structured and had three peaks with a spacing of $\sim 20$ months between the first and the third peak, but CR had two peaks (actually depressions) with a much larger spacing of $\sim 30$ months. The first peaks of $R z$ and CR were almost simultaneous, but the lag of several months between the starting of CR modulation and sunspot minimum was not seen for the peaks. Hence, the second peak of CR occurred several months later than the last peak of $R z$. Thus, the gaps between the peaks did not match.

- The Tilt angle had the same type of variation as $R z$. Since $R z$ and other similar radiations do not affect the heliosphere, these are irrelevant for CR modulation. But Tilt angle in interplanetary space is very much relevant for $\mathrm{CR}$ modulation. The similarity between the variations of $R z$ and the Tilt angle implies a discrepancy (very different spacings) between the variations of Tilt angle (20 months) and $\mathrm{CR}$ variations (30 months).

- The open magnetic flux (a few solar radii above the photosphere) had a two-peak structure and the CR two-peak structure can be considered as a delayed 
effect of the open magnetic flux but for the fact that the spacings were different (flux, $\sim 25$ months; CR, $\sim 30$ months).

- The total interplanetary magnetic field $\boldsymbol{B}$ has only one peak and the $B$ variations were dissimilar to CR variations. Hence, $B$ measurements near the Earth are no good proxies for conditions in the deep heliosphere, where most of the CR modulation takes place

- Magnetic fields at Voyager 1 had a two-peak structure similar to open magnetic flux, with a spacing of $\sim 25$ months, a few months lesser than the 30 months of CR.

- The co-rotating interplanetary regions (CIR), more frequent in the declining phase of the solar cycle, had one strong peak only a few months before the second CR peak. Thus, the magnetic fields of such regions might be contributing to the $\mathrm{CR}$ modulation occurring well after the sunspots started decreasing in 2003.

- Among all these parameters, the magnetic open flux and the magnetic field at Voyager 1 seem to be closest to the deep heliospheric magnetic conditions affecting CR modulation.

Cane et al. (1999) showed that the variations of the interplanetary magnetic field strength $(B)$ over a 22 -year period are tracked by the inverted profile of the cosmic ray density measured by neutron monitors and suggested that global changes in the Sun's magnetic field were more important for long-term CR modulation than magnetic field enhancements resulting from the merging of high-speed flows and coronal mass ejections in the outer heliosphere. We feel that CR modulation is certainly due to magnetic field structures in deep heliosphere, but the structures may not be related exclusively to solar open magnetic fields and may be related at least partly to the dissipation of CME shocks (Global MIR, GMIR) and CIR (co-rotating interplanetary regions) also, which seem to occur more often in the declining phase of a sunspot cycle.

Acknowledgements. Thanks are due to W. M. Wang, I. G. Richardson and E. Echer for supplying some data privately. Thanks are due to the referees for valuable suggestions. This work was partially supported by FNDCT, Brazil, under contract FINEP-537/CT.

Topcial Editor R. Forsyth thanks two anonymous referees for their help in evaluating this paper.

\section{References}

Alves, M. V., Echer, E., and Gonzalez, W. D.: Geoeffectiveness of corotating interaction regions as measured by $D_{s t}$ index, J. Geophys. Res. 111, A07S05, doi:10.1029/2005JA011379, 2006.

Atac, T. and Ozguc, A.: Flare index of solar cycle 22, Solar Phys., 180, 397-407, 1998.
Burlaga, L. F., McDonald, F. B., Goldstein, M. N., and Lazarus, A. J.: Cosmic ray modulation and turbulent interaction regions near 11 AU, J. Geophys. Res., 90, 12 027-12 039, 1985.

Burlaga, L. F., McDonald, F. B., and Ness, N. F.: Cosmic ray modulation and the distant heliospheric magnetic field: Voyager 1 and 2 observations from 1986 to 1989, near 11 AU, J. Geophys. Res., 98, 1-11, 1993.

Burlaga, L. F., Ness, N. F., and McDonald, F. B.: Voyagers 1 and 2 observe a GMIR and associated cosmic ray decreases at 61 and 82 AU, Proc. Int. Conf. Cosmic Rays, 27(9), 3641-3643, 2001.

Cane, H. V., Richardson, I. G., and von Rosenvinge, T. T.: Cosmic ray decreases: 1964-1994, J. Geophys. Res., 101, 21 561$21572,1996$.

Cane, H. V., Wibberenz, G., Richardson, I. G., and von Rosenvinge, T. T.: Cosmic ray modulation and the solar magnetic field, Geophys. Res. Lett., 26, 565-568, 1999.

Dorman, L. I.: To the theory of cosmic ray modulation by solar wind, Proc. 6th Int. Cosmic Ray Conf., Moscow, 4, 328-334, 1959.

Dorman, L. I.: Cosmic ray long-term variation: even-odd cycle effect, role of drifts, and the onset of cycle 23, Adv. Space Res., 27(3), 601-606, 2001.

Dorman, L. I., Dorman, I. V., Iucci, N., Parisi, M., and Villoresi, G.: Hysteresis between solar activity and cosmic rays during cycle 22: The role of drifts, and the modulation region, Adv. Space Res., 27(3), 589-594, 2001a.

Dorman, L. I., Iucci, N., and Villoresi, G.: Time lag between cosmic rays and solar activity in solar minimum 1994-1996 and the residual modulation, Adv. Space Res., 27(3), 589-594, 2001 b.

Ferreira, S. and Potgieter, M.: On the 'tilt angles' of the heliospheric current sheet used in modulation models over a 22-year cosmic ray cycle, 34th COSPAR Scientific Assembly, The Second World Space Congress, held 10-19 October 2002 in Houston, TX, USA., meeting abstract, 2002.

Harvey, K. L. and Recely, F.: Polar coronal holes during cycles 22 and 23, Solar Phys., 211, 31-52, 2002.

Hoeksema, J. T.: The Large-Scale Structure of the Heliospheric Current Sheet During the ULYSSES Epoch, Space Sci. Rev., 72, 137-148, 1995.

Jokipii, J. R. and Davila, J. M.: Effects of particle drift on the transport of cosmic rays, IV-more realistic diffusion coefficients, Astrophys. J., 248, Part 1, 1156-1161, 1981.

Jokipii, J. R. and Thomas, B.: Effects of drift on the transport of cosmic rays, IV-modulation by a wavy interplanetary current sheet, Astrophys. J., 243, Part 1, 1115-1122, 1981.

Kane, R. P.: Long-term variations of solar, interplanetary, geomagnetic indices and comic ray intensities-A brief tutorial, Ind. J. Radio Space Phys., 35, 312-323, 2006a.

Kane, R. P.: A detailed comparison of cosmic ray gaps with solar Gnevyshev gaps, Solar Phys., 236, 207-226, 2006 b.

Kane, R. P.: Modulation of cosmic rays in cycle 23: Some striking features, J. Atmos. Solar-Terres. Phys., 68, 1291-1310, 2006c.

Lee, M. A. and Fisk, L. A.: The role of particle drifts in solar modulation, Astrophys. J, 248, Part 1, 836-844, 1981.

Maris, O. and Maris, G.: High-speed plasma streams in solar wind, Romanian Rep Physics, 55, 259-269, 2003.

Parker, E. N.: Dynamic Processes in the Interplanetary Medium, Intersci. Publ., 1963.

Potgieter, M. S. and Moraal, H.: A drift model for the modulation 
of galactic cosmic rays, Astrophys. J., 294, 425-440, 1985.

Rybansky, M., Rusin, V., Minarovjech, M., and Klocok, L.: Reexamination of the coronal index of solar activity, J. Geophys. Res., 110, A08/06, doi:10.1029/2005JA011146, 2005.

Wang, Y.-M. and Sheeley, N. R.: Sunspot activity and the longterm variation of the Sun's open magnetic flux, J. Geophys. Res. 107(A10), 1302, doi:10.1029/2001JA000500, SSH 10, 1$15,2002$.

Wang, Y. M., Lean, I., and Sheeley Jr., N. R.: The long-term variation of the Sun's open magnetic flux, Geophys. Res. Lett., 27, 505-508, 2000.
Wibberenz, G., Richardson, I. G., and Cane, H. V.: A simple concept for modeling cosmic ray modulation in the inner heliosphere during solar cycles 20-23, J. Geophys. Res., 107(A11), 1353, doi:10.1029/2002JA009461, SSH 5, 1-15, 2002.

Woods, T. N., Tobiska, W. K., Rottman, G. J., and Worden, J. R.: Improved solar Lyman alpha irradiance modeling from 1947 through 1999 based on UARS observations, J. Geophys. Res., 105(A12), 27 195-27 215, 2000. 